\title{
The Association Between Allergy Skin Testing, Atopic Respiratory Conditions, and Stroke Mortality in Middle-Aged and Elderly Adults
}

\author{
Eric M. Matheson, MD, Arch G. Mainous III, PhD, and Mark A. Carnemolla, BS
}

Background: A history of atopic respiratory conditions has been linked to an increased risk of stroke. What remains unclear is whether positive allergy skin testing is associated with an increased risk of stroke. The primary goal of this study was to determine whether positive allergy skin testing is associated with an increased risk of fatal stroke. A secondary goal is to determine whether having both positive allergy skin testing and an atopic respiratory condition is associated with a particularly high risk of stroke death.

Methods: An analysis was performed of the National Health and Nutrition Examination Survey II Mortality Cohort.

Results: Controlling for age, gender, race, alcohol use, smoking status, diabetes, hypertension, and body mass index, patients with positive allergy skin testing had a hazard ratio for stroke mortality of 1.56 (95\% CI, 1.01-2.40) versus those without positive allergy testing. Patients with both positive allergy testing and an atopic respiratory condition had a hazard ratio for stroke mortality of 2.31 (95\% CI, 1.13-4.73).

Conclusions: Individuals with both positive allergy skin testing and an atopic respiratory condition have more than a 2-fold increased risk of fatal stroke. This novel risk factor has substantial implications for a large segment of the population not previously considered at risk. (J Am Board Fam Med 2009;22:604-609.)

Stroke is the third leading cause of mortality in the United States, accounting for more than 150,000 deaths in 2004. ${ }^{1,2}$ This number is expected to rise significantly as the population of the United States ages. Progress has been made in identifying the risk factors that are responsible for stroke, but our understanding remains incomplete. Even the most complex multivariate models including major risk factors such as smoking and diabetes as well as more minor risk factors fail to fully predict who is at risk for stroke. ${ }^{3}$

This article was externally peer reviewed.

Submitted 2 January 2009; revised 23 March 2009; accepted 1 April 2009.

From the Department of Family Medicine, Medical University of South Carolina, Charleston.

Funding: Supported by grant no. 5 D55HP05150 from the Health Resources and Services Administration.

Conflict of interest: none declared.

Corresponding author: Eric M. Matheson, MD, Department of Family Medicine, Medical University of South Carolina, 295 Calhoun Street, Charleston, SC 29425 (E-mail: matheson@musc.edu).
In an effort to expand the understanding of stroke risk factors, researchers have investigated the link between stroke and a number of chronic diseases. One promising area of investigation has been research examining the relationship between atopic respiratory conditions and stroke. Asthma, a disease strongly tied to atopy, was associated with a $50 \%$ increase in combined fatal and nonfatal stroke risk. ${ }^{4}$ Building on the notion that atopy may be a risk factor for stroke, we found that a history of physician-diagnosed hay fever was associated with more than a $70 \%$ increase in combined fatal and nonfatal stroke risk. ${ }^{5}$ Because hay fever may have some misclassification as an indicator of atopic sensitivity, it is unclear whether the relationship between atopic respiratory conditions and stroke exists with a more systematic measure of atopy such as allergy skin testing. To clarify this issue, we examined the risk of stroke mortality in patients with positive allergy skin testing in a nationally representative cohort. We then subdivided the group of patients with positive allergy skin testing into those with a his- 
tory of atopic respiratory conditions (hay fever and/or asthma) and those without atopic respiratory conditions.

\section{Methods}

\section{Dataset}

We analyzed data from the National Health and Nutrition Examination Survey II (NHANES II) and linked it to the NHANES II Mortality Cohort. The NHANES II is a nationally representative survey based on a complex sampling design that allows for weighted population estimates of the noninstitutionalized United States population. The NHANES II was conducted in the United States between February 1976 and February 1980. The sample was composed of approximately 27,801 noninstitutionalized United States civilians, with an age range of 6 months to 74 years, recruited at 64 sampling sites. The survey included a physical examination, numerous laboratory studies, allergy skin testing, and an extensive questionnaire about lifestyle, diet, and medical history. A thorough description of the NHANES II survey and sampling procedure has been described previously. ${ }^{6}$

The NHANES II Mortality Study was a prospective cohort study comprising 9252 adults between the ages of 30 and 75 and done at the time of the original NHANES II study. ${ }^{7}$ Deaths were captured by linking information from the National Death Index to matching personal identifiers in the NHANES II. Based on previous studies, more than $90 \%$ of all deaths can be identified by using this method. ${ }^{8,9}$ In our study, we followed patients 45 years of age and older from 1976 through December 31, 1992 (Table 1). We did not include patients younger than 45 years of age because the pathophysiology of strokes in that population differs significantly from those older than age 45 . In individuals $\leq 45$ years of age, $42.7 \%$ of all strokes are either subarachnoid or intracerebral hemorrhages, whereas in individuals $>45$ years of age these comprise only $15.7 \%$ of strokes. ${ }^{10}$

\section{Atopic Respiratory Conditions}

Individuals were considered to have an atopic respiratory condition if a physician had ever diagnosed them with asthma, hay fever, or both asthma and hay fever.
Table 1. Characteristics of participants $\geq 45$ Years of Age in the National Health and Nutrition Examination Survey II Mortality Study at Baseline*

\begin{tabular}{|c|c|}
\hline \multicolumn{2}{|l|}{ Participants (n) } \\
\hline Unweighted & 5119 \\
\hline Weighted (million) & 45.8 \\
\hline \multicolumn{2}{|l|}{ Allergy skin testing (\%) } \\
\hline Negative & 77.0 \\
\hline Positive & 23.0 \\
\hline \multicolumn{2}{|l|}{ Sex $(\%)$} \\
\hline Male & 45.5 \\
\hline Female & 54.5 \\
\hline \multicolumn{2}{|l|}{ Race (\%) } \\
\hline Non-Hispanic white & 86.2 \\
\hline Non-Hispanic black & 9.9 \\
\hline Hispanic & 3.9 \\
\hline \multicolumn{2}{|l|}{ Smoking status (\%) } \\
\hline Nonsmoker & 63.7 \\
\hline Current & 36.3 \\
\hline \multicolumn{2}{|l|}{ Diabetes (\%) } \\
\hline No & 94.6 \\
\hline Yes & 5.4 \\
\hline \multicolumn{2}{|l|}{ High blood pressure (\%) } \\
\hline No & 65.4 \\
\hline Yes & 34.6 \\
\hline \multicolumn{2}{|l|}{ Alcohol use (\%) } \\
\hline Nondrinker & 39.4 \\
\hline Drinker & 60.6 \\
\hline \multicolumn{2}{|l|}{ LDL cholesterol (\%) } \\
\hline$<160 \mathrm{mg} / \mathrm{dL}$ & 84.6 \\
\hline$\geq 160 \mathrm{mg} / \mathrm{dL}$ & 15.4 \\
\hline \multicolumn{2}{|l|}{ Physical activity (\%) } \\
\hline Sedentary & 10.9 \\
\hline Low & 50.4 \\
\hline High & 38.7 \\
\hline Mean age $(\mathrm{SD})$ & $57.4(0.14)$ \\
\hline Mean body mass index $\left(\mathrm{kg} / \mathrm{m}^{2}\right)(\mathrm{SD})$ & $26.3(0.11)$ \\
\hline
\end{tabular}

*This excludes individuals with a history of myocardial infarction, congestive heart failure, or stroke.

LDL, low-density lipoprotein.

\section{Allergy Testing}

For the purposes of our analysis, all patients who reported a history of positive allergy skin testing were included in the group with positive allergy skin testing. Those who did not report a history of positive allergy skin testing underwent allergy skin testing and were included in the positive allergy skin testing group if they had a positive allergy response to one or more of the tested allergens. Allergy testing was performed using a traditional "skin prick test" administered in a sterile fashion with a 25 gauge, 3/8-inch needle. The allergens 
tested included Alternaria (a common fungus), Bermuda grass, cat, dog, house dust, mixed long and short ragweed, oak, and perennial rye grass. Histamine served as the control. After administration of the allergens, readings for wheal and flare reactions were made at 10 minutes and again at 20 minutes. ${ }^{11}$ For the purposes of our analysis, we considered a test positive if a wheal and flare reaction occurred or if a flare reaction of greater than $5 \mathrm{~mm}$ developed with any of the allergens tested.

\section{Stroke Mortality}

Stroke mortality was determined using death certificates through the National Death Index. We used the International Classification of Disease Ninth Revision codes 430 to 438 for stroke mortality.

\section{Control Variables}

Several control variables have been identified as risk factors for stroke mortality and thus may be potential cofounders of any relationship between positive allergy skin testing and stroke mortality. ${ }^{12-17}$ Control variables in our study included age, sex, race, body mass index (body weight in $\mathrm{kg} /$ height in $\mathrm{m}^{2}$ ), hyperlipidemia (defined as low-density lipoproteins $\geq 160$ $\mathrm{mg} / \mathrm{dL}$ ), diabetes, hypertension, and smoking. Age and body mass index were treated as continuous variables. Sex, hyperlipidemia, diabetes, hypertension, and smoking were treated as categorical variables. Hyperlipidemia was defined as a low-density lipoprotein level $\geq 160 \mathrm{mg} / \mathrm{dL}$, and patients were categorized as either being hyperlipidemic or nonhyperlipidemic. Diabetes was determined by self-report and patients were categorized as either diabetic or nondiabetic. Hypertension and smoking were also categorized by self-report and patients were categorized as either hypertensive or normotensive and as either a current smoker or current nonsmoker, respectively.

\section{Analysis}

Because of the complex sampling design of the NHANES II, we used the statistical packages SUDAAN (SUDAAN Statistical Software Center, Research Triangle Park, NC) and SAS (SAS Inc., Cary, NC) to perform all analyses. We excluded participants with a history of stroke or coronary vascular disease because of their extremely high risk of death from recurrent stroke. ${ }^{17} \mathrm{We}$ used $\chi^{2}$ analysis to examine the bivariate relationships between participants with positive allergy skin testing and those with negative allergy skin testing for each of the categorical control variables. $T$ tests were computed to examine the relationship between participants with positive allergy skin testing and those with negative allergy skin testing for each of the continuous control variables. We used a Cox proportional hazards regression to model time to stroke mortality, adjusting for age only. We used age as a covariate (1) because the prevalence of positive allergy skin testing decreases with age and (2) because stroke prevalence increases with age. ${ }^{18,19}$ For example, in our current dataset, if we compare patients aged 45 to 54 to those aged 65 to 74 , the incidence of stroke increases by more than $850 \%$, whereas the incidence of positive allergy skin testing decreases by $25 \%$. Because of this relationship, if we fail to adjust for age we confound any relationship that may exist between positive allergy skin testing and stroke. After completing the preliminary regression we performed a fully adjusted regression with age, sex, race, body mass index, diabetes, hypertension, smoking, and hyperlipidemia as covariates. Because we were using a Cox proportional hazards analysis, we conducted a Schoenfeld's test to determine whether the hazards were proportional. ${ }^{20}$ After performing our primary analysis examining the relationship between positive allergy skin testing and risk of fatal stroke, we performed 2 further survival analyses using the same cohort. In the first analysis we performed a Cox proportional hazards analysis comparing the risk of fatal stroke for individuals with a reported history of respiratory atopy versus those without a history of respiratory atopy. We did not include allergy skin testing in this analysis. In the second analysis we performed a Cox proportional hazards analysis comparing the risk of fatal stroke for individuals with both a history of respiratory atopy and positive allergy skin testing versus the remainder of the cohort.

\section{Results}

Our cohort consisted of a unweighted sample of 5119 eligible participants (weighted, 45.8 million) at baseline with a mean follow-up of 11.2 years and an average age of 57.4 years. There were 119 stroke deaths (weighted, 732,363 stroke deaths) during follow-up, with 30 stroke deaths (weighted, 202,081 stroke deaths) in the group with positive allergy skin testing and 89 stroke deaths (weighted, 
530,282 stroke deaths) in the group without positive allergy skin testing. Participants with positive allergy skin testing comprised $23.0 \%$ of the cohort. In bivariate relationships, age and alcohol use were significantly different between the group with positive allergy skin testing and the group with negative allergy skin testing. Individuals with positive allergy skin testing were approximately 1 year younger than those with negative allergy skin testing and a higher percentage consumed alcohol (66.1\% in the group with positive allergy skin testing versus $59.0 \%$ in the group with negative allergy skin testing).

In a survival analysis adjusting for age, patients with positive allergy skin testing had a hazard ratio (HR) for stroke mortality of 1.45 (95\% CI, 0.932.25 ) versus those with negative allergy skin testing. A statistically significant relationship between positive allergy testing and risk of stroke mortality was found in a fully adjusted model (HR, 1.56; 95\% CI, 1.01-2.40), controlling for relevant confounding variables (Table 2). In a survival analysis adjusting for age, patients with a self-reported history of respiratory atopy had an HR for stroke mortality of 1.45 (95\% CI, 0.76-2.80) versus those without a history of respiratory atopy. In a fully adjusted model, patients with a self-reported history of respiratory atopy had an HR for stroke mortality of 1.52 (95\% CI, 0.80- 2.92), controlling for relevant confounding variables (Table 2). In a survival analysis of individuals with both positive allergy skin testing and a history of respiratory atopy, we found an HR for stroke mortality of 2.11 (95\% CI, 1.024.37) versus the remainder of the cohort, controlling for age. In a fully adjusted model, patients with both positive allergy skin testing and a history of respiratory atopy had an HR for stroke mortality of 2.31 (95\% CI, 1.13-4.73), controlling for relevant confounding variables (Table 2).

Schoenfeld testing indicated that the hazards were proportional in all of the survival analyses that were performed.

\section{Discussion}

The results of this study support previous works suggesting a relationship between atopic respiratory conditions and increased stroke risk. The current findings indicate that individuals with a history of both respiratory atopy and positive allergy skin testing could have more than a 2 -fold increased risk
Table 2. Cox Proportional Hazards Models for Risk of Fatal Stroke in Participants with Positive Allergy Skin Testing, a History of Respiratory Atopy, or Both*

\begin{tabular}{|c|c|c|}
\hline & Hazard Ratio & $95 \% \mathrm{CI}$ \\
\hline \multicolumn{3}{|c|}{ Positive allergy skin testing } \\
\hline \multicolumn{3}{|c|}{ Age-adjusted model } \\
\hline No & 1.00 & - \\
\hline Yes & 1.45 & $0.93-2.25$ \\
\hline \multicolumn{3}{|c|}{ Fully adjusted model $^{\dagger}$} \\
\hline No & 1.00 & - \\
\hline Yes & 1.56 & $1.01-2.40$ \\
\hline \multicolumn{3}{|c|}{ History of respiratory atopy } \\
\hline \multicolumn{3}{|c|}{ Age adjusted model } \\
\hline No & 1.00 & - \\
\hline Yes & 1.45 & $0.76-2.80$ \\
\hline \multicolumn{3}{|c|}{ Fully adjusted Model $^{\dagger}$} \\
\hline No & 1.00 & - \\
\hline Yes & 1.52 & $0.80-2.92$ \\
\hline \multicolumn{3}{|c|}{$\begin{array}{l}\text { Positive allergy skin testing } \\
\text { and a history of } \\
\text { respiratory atopy }\end{array}$} \\
\hline \multicolumn{3}{|c|}{ Age adjusted model } \\
\hline No & 1.00 & - \\
\hline Yes & 2.11 & $1.02-4.37$ \\
\hline \multicolumn{3}{|c|}{ Fully adjusted model ${ }^{\dagger}$} \\
\hline No & 1.00 & - \\
\hline Yes & 2.31 & $1.13-4.73$ \\
\hline
\end{tabular}

*This excludes individuals with a history of myocardial infarction, congestive heart failure, or stroke. A history of respiratory atopy was defined as having been diagnosed by a physician with asthma, hay fever, or both at any time in the past.

${ }^{\dagger}$ Adjusted for age, sex, race, smoking status, diabetes, hypertension, body mass index, alcohol consumption, and low-density lipoprotein cholesterol $\geq 160 \mathrm{mg} / \mathrm{dL}$.

of fatal stroke. It is significant that individuals who have both respiratory atopy and positive allergy skin testing are at much higher risk than those with either a history of respiratory atopy or positive allergy skin testing alone. Given that $23 \%$ of our cohort had positive allergy skin testing to one or more common allergens, this study's findings may have broad public health implications regarding who may be at increased risk of stroke death.

Why might atopic respiratory conditions be a risk factor for future stroke? One potential explanation is that atopic respiratory conditions could exacerbate sleep apnea by increasing airway resistance through swelling of the respiratory mucosa. ${ }^{21}$ This may be important because sleep apnea is a noteworthy risk factor for stroke. ${ }^{22}$

Another possibility is that atopic respiratory conditions may increase the risk of stroke by in- 
creasing blood pressure. ${ }^{23,24}$ Although our analysis suggests that the association between atopic respiratory conditions and stroke remains even after controlling for a self-reported history of hypertension, blood pressure may still be important because, we suspect, atopic respiratory conditions raise blood pressure only intermittently during acute allergy attacks, which could easily be missed with typical blood pressure screenings. We also suspect that atopic respiratory conditions contribute to the so-called "upper airway resistance syndrome," which can cause increased blood pressure during sleep in otherwise normotensive individuals. ${ }^{25} \mathrm{We}$ believe it is important to emphasize that patients can have upper airway resistance syndrome without meeting the formal definition of sleep apnea. ${ }^{26}$

During the past 2 decades, increasing evidence has linked variations in the expression of inflammatory mediators, such as interleukin (IL)-6, IL-10, and 5-lipoxygenase, to stroke. ${ }^{27-32}$ Atopic respiratory conditions have also been linked to similar changes in IL-6, IL-10, and 5-lipoxygenase. ${ }^{3-36}$ Given these relationships, inflammation may be a critical link between atopic respiratory conditions and stroke.

This study has a few limitations of note. Stroke death was determined using death certificates rather than through direct imaging; however, because the attending physician is responsible for death certificate completion, he or she would presumably know how the patient died. In addition, participants who reported a history of positive allergy skin testing did not undergo allergy skin testing but were included in the allergy group. Because repeating previously positive skin allergy testing was not performed in the NHANES II, we included in the positive allergy skin testing group those individuals who stated that they had a previous positive skin test despite the lack of independent confirmatory testing. It is possible that this could overestimate the true number of individuals with positive allergy skin testing, but because misrepresentation of a previous positive skin test is not likely to be related to future stroke, we do not feel that this would have influenced our results. In addition, we hoped to investigate the relationship between allergies and stroke type (ischemic versus hemorrhagic), but we lacked sufficient power to perform this analysis. Finally, we were unable to determine how the use of antihistamines to treat respiratory atopy may be related to the risk of stroke because the NHANES II did not clearly delineate antihistamines from other allergy medications such as decongestants.

\section{Conclusions}

Having both a history of respiratory atopy and positive allergy skin testing seems to be a risk factor for stroke mortality. This relatively novel risk factor has substantial implications for a large segment of the population not previously considered to be at risk. We believe additional research is merited to further explore these findings and determine whether allergy medications such as antihistamines modify stroke risk in those with an atopic respiratory condition.

\section{References}

1. Heron M. Deaths: leading causes for 2004. Natl Vital Stat Rep 2007;56:1-95.

2. Rosamond W, Flegal K, Friday G, et al. Heart disease and stroke statistics-2007 update: a report from the American Heart Association Statistics Committee and Stroke Statistics Subcommittee. Circulation 2007;115:e69-171.

3. Chambless LE, Heiss G, Shahar E, Earp MJ, Toole J. Prediction of ischemic stroke risk in the Atherosclerosis Risk in Communities Study. Am J Epidemiol 2004;160:259-69.

4. Schanen JG, Iribarren C, Shahar E, et al. Asthma and incident cardiovascular disease: the Atherosclerosis Risk in Communities Study. Thorax 2005;60: 633-8.

5. Matheson EM, Player MS, Mainous AG 3rd, King DE, Everett CJ. The association between hay fever and stroke in a cohort of middle-aged and elderly adults. J Am Board Fam Med 2008;21:179-83.

6. McDowell A, Engel A, Massey JT, Maurer K. Plan and operation of the Second National Health and Nutrition Examination Survey, 1976-1980. Vital Health Stat 1. 1981;15:1-144.

7. Loria CM, Sempos CT, Vuong C. Plan and operation of the NHANES II Mortality Study, 1992. Vital Health Stat 1 1999;38:1-16.

8. Stampfer MJ, Willett WC, Speizer FE, et al. Test of the National Death Index. Am J Epidemiol 1984; 119:837-9.

9. Williams BC, Demitrack LB, Fries BE. The accuracy of the National Death Index when personal identifiers other than Social Security number are used. Am J Public Health 1992;82:1145-7.

10. Marini C, Totaro R, De Santis F, Ciancarelli I, Baldassarre M, Carolei A. Stroke in young adults in the community-based L'Aquila registry: incidence and prognosis. Stroke 2001;32:52-6.

11. National Health and Nutrition Examination Survey. 
Public use data tape documentation: allergy skin testing tape 5309. National Health and Nutrition Examination Survey, 1976-1980:101-5.

12. Seshadri S, Beiser A, Kelly-Hayes M, et al. The lifetime risk of stroke: estimates from the Framingham Study. Stroke 2006;37:345-50.

13. Centers for Disease Control and Prevention. Agespecific excess deaths associated with stroke among racial/ethnic minority populations-United States, 1997. JAMA 2000;283:2382-3.

14. Ueshima H, Choudhury SR, Okayama A, et al. Cigarette smoking as a risk factor for stroke death in Japan: NIPPON DATA80. Stroke 2004;35:183641.

15. Almdal T, Scharling H, Jensen JS, Vestergaard H. The independent effect of type 2 diabetes mellitus on ischemic heart disease, stroke, and death: a population-based study of 13,000 men and women with 20 years of follow-up. Arch Intern Med 2004;164: 1422-6.

16. Hansagi H, Romelsjo A, Gerhardsson de Verdier M, Andreasson S, Leifman A. Alcohol consumption and stroke mortality. 20-year follow-up of 15,077 men and women. Stroke. 1995;26:1768-73.

17. Straus SE, Majumdar SR, McAlister FA. New evidence for stroke prevention: clinical applications. JAMA 2002;288:1396-8.

18. Gergen PJ, Turkeltaub PC, Sempos CT. Is allergen skin test reactivity a predictor of mortality? Findings from a national cohort. Clin Exp Allergy 2000;30: 1717-23.

19. Wolf PA, D'Agostino RB, Belanger AJ, Kannel WB. Probability of stroke: a risk profile from the Framingham Study. Stroke 1991;22:312-8.

20. Schoenfeld D. Partial residuals for the proportional hazards regression model. Biometrika 1982;69:23941.

21. Braunstahl GJ, Fokkens WJ, Overbeek SE, KleinJan A, Hoogsteden HC, Prins JB. Mucosal and systemic inflammatory changes in allergic rhinitis and asthma: a comparison between upper and lower airways. Clin Exp Allergy 2003;33:579-87.

22. Yaggi HK, Concato J, Kernan WN, Lichtman JH, Brass LM, Mohsenin V. Obstructive sleep apnea as a risk factor for stroke and death. $\mathrm{N}$ Engl J Med 2005;353:2034-41.

23. Magen E, Yosefy C, Viskoper RJ, Mishal J. Treatment of allergic rhinitis can improve blood pressure control. J Hum Hypertens 2006;20:888-93.

24. Kony S, Zureik M, Neukirch C, Leynaert B, Vervloet D, Neukirch F. Rhinitis is associated with in- creased systolic blood pressure in men: a populationbased study. Am J Respir Crit Care Med 2003;167: $538-43$.

25. Guilleminault C, Stoohs R, Shiomi T, Kushida C, Schnittger I. Upper airway resistance syndrome, nocturnal blood pressure monitoring, and borderline hypertension. Chest 1996;109:901-8.

26. Guilleminault C, Chowdhuri S. Upper airway resistance syndrome is a distinct syndrome. Am J Respir Crit Care Med 2000;161:1412-3.

27. Hoshi T, Kitagawa K, Yamagami H, Furukado S, Hougaku H, Hori M. Relation between interleukin-6 level and subclinical intracranial large-artery atherosclerosis. Atherosclerosis 2008;197:326-32.

28. Cesari M, Penninx BW, Newman AB, et al. Inflammatory markers and onset of cardiovascular events: results from the Health ABC study. Circulation 2003;108:2317-22.

29. van Exel E, Gussekloo J, de Craen AJ, Bootsma-van der Wiel A, Frolich M, Westendorp RG. Inflammation and stroke: the Leiden 85-Plus Study. Stroke 2002;33:1135-8.

30. Mallat Z, Besnard S, Duriez M, et al. Protective role of interleukin-10 in atherosclerosis. Circ Res 1999; $85:$ e17-24.

31. Cipollone F, Mezzetti A, Fazia ML, et al. Association between 5-lipoxygenase expression and plaque instability in humans. Arterioscler Thromb Vasc Biol 2005;25:1665-70.

32. Klemens C, Rasp G, Jund F, et al. Mediators and cytokines in allergic and viral-triggered rhinitis. Allergy Asthma Proc 2007;28:434-41.

33. Krishnamoorthy N, Oriss TB, Paglia M, et ak. Activation of c-Kit in dendritic cells regulates $\mathrm{T}$ helper cell differentiation and allergic asthma. Nat Med 2008;14:565-73.

34. Heaton T, Rowe J, Turner S, et al. An immunoepidemiological approach to asthma: identification of in-vitro T-cell response patterns associated with different wheezing phenotypes in children. Lancet 2005;365:142-9.

35. Enrique E, Pineda F, Malek T, et al. Sublingual immunotherapy for hazelnut food allergy: a randomized, double-blind, placebo-controlled study with a standardized hazelnut extract. J Allergy Clin Immuno 2005;116:1073-9.

36. Allayee H, Hartiala J, Lee $W$, et al. The effect of montelukast and low-dose theophylline on cardiovascular disease risk factors in asthmatics. Chest 2007; 132:868-74. 